\title{
PATHOPHYSIOLOGICAL CORRELATIONS BETWEEN ANKYLOSING SPONDYLITIS AND PERIODONTAL DISEASE. THE IMPACT OF ANKYLOSING SPONDYLITIS THERAPY ON PERIODONTAL TISSUE
}

\author{
Alexandru G. Croitoru ${ }^{1,2}$, Dan Piperea-Sianu ${ }^{1,3}$, Carina Mihai ${ }^{1,4}$, Alice Piperea-Sianu ${ }^{5}$, \\ Adela Maria Ceau ${ }^{1}$, Daniela Badita ${ }^{1,3}$, Victoria Arama ${ }^{1,6}$, Stefan Sorin Arama ${ }^{1,2}$ \\ ${ }^{1}$ Carol Davila University of Medicine and Pharmacy, Bucharest \\ ${ }^{2}$ Faculty Of Dental Medicine, Department of Physiopathology and Immunology \\ ${ }^{3}$ Faculty of Dental Medicine, Department of Physiology \\ ${ }^{4}$ Faculty of Medicine, Department of Internal Medicine and Rheumatology, Cantacuzino Hospital \\ ${ }^{5}$ Titu Maiorescu University, Department of Analytic Chemistry \\ ${ }^{6}$ Faculty of Medicine, Matei Bals National Institute of Infectious Diseases
}

\begin{abstract}
Periodontal disease (PD) and ankylosing spondylitis (AS) are both chronic inflammatory conditions characterized by a destructive process in the deep marginal periodontal tissue and, respectively, in the joints and entheses. This article summarizes all current data regarding the association between PD and AS and assesses the impact of AS therapy on periodontal tissue.
\end{abstract}

Keywords: ankylosing spondylitis, periodontal disease, TNF-a inhibitors

\section{INTRODUCTION}

Periodontal disease (PD) is a pathological concept that brings together inflammatory, proliferative and degenerative changes of marginal periodontal tissue. PD has a chronic course, generally without noisy symptoms to determine the patient's presentation to the doctor, which explains the increased morbidity and delayed addressing to the doctor, in final evolutionary stages. Marginal periodontal tissue changes are widespread globally and include all age groups and geographic regions. Due to the large variety of clinical forms of $\mathrm{PD}$, data on prevalence varies between $10-15 \%$ for medium and severe forms. PDs etiology is likely microbial, involving germs grouped in microbial complexes according to virulence and the order they occur in the evolutionary process. Contributing factors are tartar, morphological changes of teeth and dental arches, temporomandibular joint disorders, occlusal trauma, and systemic factors. They act by enabling plaque accumulation (Fig. 1) and intimate contact between it and marginal periodontium (tartar, incongruence by dental crowding), by inadequate periodontal stress (occlusal trauma) or by reducing defense capacity and increasing susceptibility to infection (systemic diseases). Untreated, PD evolves from superficial form of marginal periodontal illness (gum and supra-alveolar ligaments) to deep form, meaning irreversible destruction of the alveolar bone and periodontal ligament with loss of support tissue (Fig. 2) and spontaneous avulsion of teeth from the alveoli $(1,2)$.

\section{Correspondence address:}

Alexandru G. Croitoru, MD, PhD, Assistant Professor, Faculty Of Dental Medicine, Department of Physiopathology and Immunology, Carol Davila Unversity of Medicine and Pharmacy, Dionisie Lupu St. 37, 1st District, 020022 Bucharest

E-mail: acroitoru@cnmv.ro 


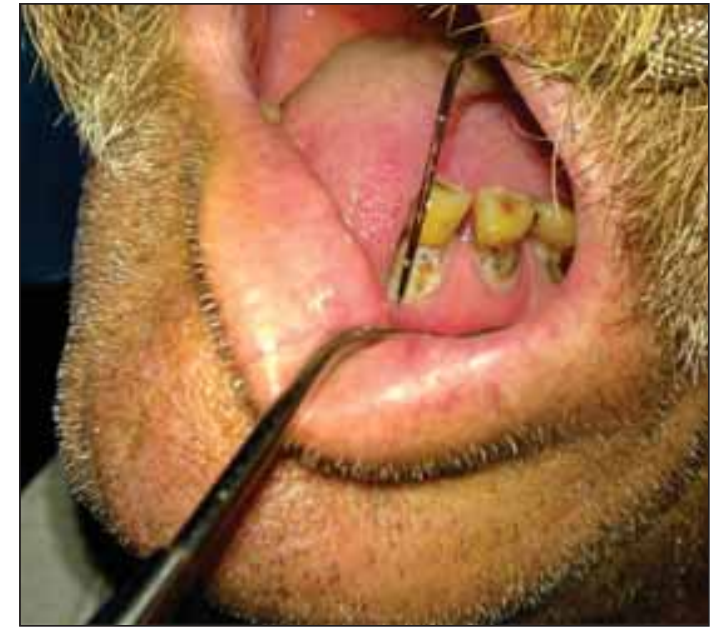

FIGURE 1. Patient with gingival retraction, root tartar and plaque (courtesy of Dan Piperea-Sianu, MD)

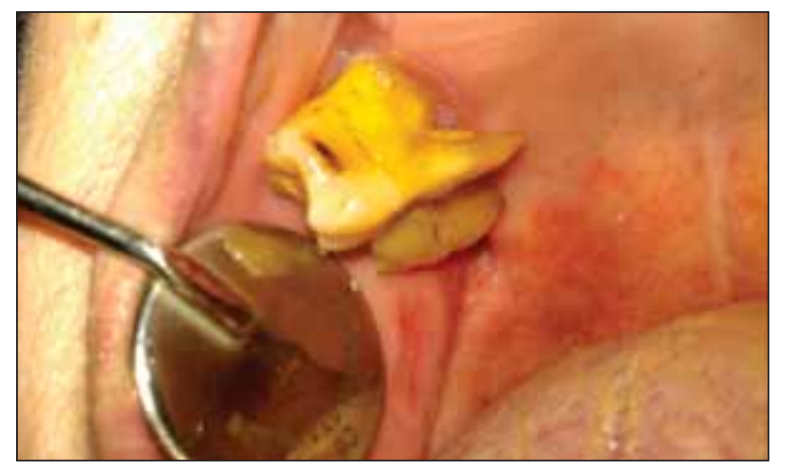

FIGURE 2. Patient with severe PD. Molars with advanced bone resorption and grade 3 mobility (courtesy of Dan Piperea-Sianu, MD)

Spondyloarthropathies are a group of inflammatory arthropathies different from rheumatoid arthritis (RA), which include ankylosing spondylitis, psoriatic arthritis, reactive arthritis and enteropathic arthropathies (associated with Crohn's disease or ulcerative colitis). Ankylosing spondylitis (AS) is the main representative of the class. AS is a chronic inflammatory disease that predominantly affects the spine, starts from sacroiliac joint, has an upward trend and tendency to fibrosis, ossification and eventually stiffening of the spine. Symptoms include morning stiffness, fatigue and chronic morning and/ or nocturnal back pain. Etiology is unknown and pathogenesis is still incompletely understood, but the most important role is assigned to the HLA-B27 phenotype that is found in $90-95 \%$ of patients. An apparently secondary role is played by non-HLA genetic factors (single nucleotide polymorphisms in aminopeptidase-1 - enzyme involved in the processing of antigenic peptides, preceding expression on MHC molecules), an altered nonspecific immune response and a molecular mechanism of bone forma- tion in relation to inflammation. Prevalence and incidence of AS varies in terms of geography, but global average prevalence lies around $1 \%$ with an incidence of 8 new cases per 100,000/year, and more frequently in men than in women $(5: 1)(3,4)$.

\section{PERIODONTAL DISEASE - ETIOPATHOGENIC DATA}

The destructive process of PD is initiated by bacterial lipopolysaccharide and continued by inflammatory immune response of the host. Microorganisms such as $P$. gingivalis, $T$. denticola, $T$. forsithensia of the red periodontal complex, together with A. actinomycetemcomitans serotype B produce enzymes that degrade extracellular matrix and cell membranes, in order to obtain necessary components for the synthesis of bacterial parts and tissue invasion. This triggers a local immune response leading to the release of pro-inflammatory cytokines, metalloproteinases (MMP), prostaglandins and enzymes that destroy the collagen to facilitate the migration of leukocytes to the site of inflammation. As PD progresses, collagen fibers are destroyed and the depth of periodontal pockets increases by apical migration of the epithelial insertion, exposing the alveolar bone, which leads to activation of osteoclasts and bone lysis (5).

\section{The relationship between BP and systemic health condition}

Oral health is an integral part of systemic health and the relationship between the two is currently intensively studied, providing valuable information indicating that PD could be a risk factor for various systemic diseases such as cardiovascular disease (CVD) and diabetes mellitus (DM), most likely due to high serum levels of circulating bacterial components such as endotoxins. Numerous clinical and epidemiological studies in recent years have provided valuable data on the negative impact of periodontal damage on overall health due to increased serum levels of proinflammatory cytokines, chemokines and various other mediators of inflammation. For this reason, patients with PD can be considered systemically compromised, even in the absence of signs/symptoms of other diseases (5).

\section{Bacteriemia and focal infection - PD issues}

It is possible for the oral cavity to become a remote releasing site for pathogens, particularly in 
immuno-compromised patients such as those with rheumatic diseases, malignancies, diabetes, corticosteroid or immunosuppressive therapy. Current epidemiological studies show that oral infectious sites, especially septic inflammatory processes of marginal and apical periodontal tissue are most likely to become starting points for systemic bacterial dissemination, a process facilitated by close anatomical relationship between oral microbiocenosis and bloodstream (5).

\section{AS - PD RELATIONSHIP: EPIDEMIOLOGICAL DATA}

Both AS and PD are conditions characterized by the disregulation of the host's inflammatory response, leading to damage or even loss of hard and soft support tissue. AS was correlated with other inflammatory diseases but its relation to PD is only beginning to be studied. Multiple studies support the role of PD in maintaining a biological inflammatory syndrome within a variety of chronic diseases, including AS, in which the inflammatory process is essential. It is believed that patients with AS are more likely to have PD compared to the healthy population. Epidemiological data provided by the literature on PD and AS indicate a similarity in the profiles of the two groups of patients $(6,7)$.

Analyzing the correlations between PD and AS, there were attemps to determine the prevalence of PD in patients with AS, given the high prevalence of $\mathrm{PD}$ in the general population. Pischon et al examined the association between AS and PD in a prospective case-control study including 48 patients with AS and 48 clinically healthy individuals. A detailed periodontal examination was performed in all subjects, inluding probing pocket depth, epithelial insertion level (clinical attachment loss), plaque index and bleeding on probing. Potential risk factors for PD, such as smoking, alcohol, high body mass index and education, were assessed by questionnaires. In stepwise logistic regression, including AS status, age, gender, education, smoking, alcohol consumption and BMI, only age, level of education and AS status were found to be significant predictors of PD. Among patients with AS, PD prevalence (defined as periodontal pockets deeper than $3 \mathrm{~mm}$ ) was found to be 6.81 times higher compared to healthy individuals, decreasing to 5.48 after adjusting for plaque index but remaining statistically relevant (6).

Besides prevalence, a qualitative analysis is required regarding the differences between the degree of oral damage in patients who associate the two pathologies compared to PD-only patients. In a prospective case-control study including 48 patients with AS and 48 clinically healthy individuals, Sezer et al have tried to determine the existence of a possible relationship between periodontal damage and AS evaluating serum levels of cytokines and common clinical periodontal parameters. They reached partially conclusive results, unlike the aforementioned study. Periodontal parameters, serum levels of C-reactive protein (CRP), tumor necrosis factor alpha (TNF- $\alpha$ ) and interleukin-6 (IL-6) were determined in all subjects. No statistically significant difference was observed concerning the prevalence of $\mathrm{PD}$ in patients with AS and in the control group. Furthermore, no significant differences were found in terms of depth of periodontal pockets, CAL and plaque index, the only significant clinical difference between groups being the level of bleeding on probing, which were higher in AS patients. As expected, serum markers of inflammation (CRP, TNF- $\alpha$, IL-6) showed significantly higher values in the group of AS in comparison to the control group (8).

Recently, Keller et al (Taiwan, 2013) have developed a larger, case-control study using the Longitudinal Health Insurance Database 2000 (LHIDB 2000), a database created and released to the public by the National Health Research Institute of Taiwan, which includes all the original claims data and registration files for 1 million individuals randomly sampled from the 2000 Registry for Beneficiaries of the Taiwan National Health Insurance (NHI) program (7). All patients who received a first-time diagnosis of AS from the LHIDB $2000(n=6821)$ were compared with age-, gender-, and urbanization levelmatched control subjects $(n=34,105)$ selected from the same database and who had never received a diagnosis of AS, RA, or systemic lupus erythematosus since the initiation of the NHI program in 1995. In all AS patients and controls, a history of chronic PD was identified based on the ICD-9-CM code 532.4; only patients who had at least 2 principal diagnoses of chronic PD prior to the AS diagnosis were included. The authors observed that the frequency of chronic PD is higher in patients diagnosed with AS than in the control group $(41.5 \%$ vs. $25.9 \%$ respectively), $\mathrm{p}<0.001$ by chi-square test. Correspondingly, conditional logistic regression analysis (conditioned on sex, age group, urbanization level, and index year) suggested that AS patients were more likely than controls to have been previously diagnosed as 
having chronic PD (odds ratio 1.84 [95\% confidence interval 1.74-1.98], $\mathrm{p}<0.001)$ after adjusting for monthly income and geographic location. Also, patients diagnosed with AS had a 1.7 times higher probability than controls to have undergone gingivectomy or periodontal flap surgery in the previous 5 years. Analyzing the frequency of chronic PD occurring within 1 year following a diagnosis of AS among AS patients selected in the years of 2001 and 2009, PD incidence rates were $3.3 \%$ and $2.9 \%$ respectively, a difference that was not significant (7). To conclude, this study revealed an association between AS and a prior diagnosis of chronic PD, thus confirming the results of the smaller, cross-sectional studies suggesting this association. Further studies are needed to identify an ethiologic or pathogenic link between chronic PD and the developing of AS.

\section{PERIODONTAL IMPACT OF ANTI-TNF- $\alpha$ THERAPY}

TNF- $\alpha$ is a main cytokine in pathophysiological processes like rheumatic diseases and periodontal disease. In both conditions, TNF- $\alpha$ contributes to the stimulation of osteoclasts and inhibition of osteoblasts. TNF- $\alpha$ is mainly produced by macrophages in response to the presence of antigens (lipopolysaccharide) and is present in serum and tissue inflammatory infiltrat. Its concentration correlates with disease activity score and the degree of tissue destruction. In addition to involvement in the process of osteolysis, TNF- $\alpha$ induces expression of other inflammatory mediators (prostaglandins), increases the phagocytic power of macrophages and stimulates the production of lytic enzymes (collagenase) (9).

Currently there are no studies in the literature to investigate the anti-TNF- $\alpha$ therapy in patients that suffer from PD and AS, but there is data regarding the association between periodontal damage and rheumatoid arthritis.

TNF- $\alpha$ inhibitors lead to better periodontal and joint status. Mayer et al have proposed to evaluate the influence of anti-TNF- $\alpha$ on marginal periodontium in patients with joint damage and the association between TNF- $\alpha$ from the crevicular fluid and inflammatory periodontal parameters. Regimen used in the test group was Infliximab $200 \mathrm{mg}$ every 8 weeks for $26 \pm 8$ months in addition to standard NSAIDs and DMARDs medication. The results showed: (a) gingival index significantly higher in patients without anti-TNF- $\alpha$ therapy, (b) plaque index without significant variation, (c) clinical attachment level lower in patients with TNF- $\alpha$ (d) smaller probing depth. Analysis of TNF- $\alpha$ from the crevicular fluid was performed using ELISA and aimed to find the effects of anti-TNF- $\alpha$ therapy at this level. The results of ELISA analysis revealed a lower concentration of crevicular TNF- $\alpha$ in patients under biological therapy. Also there was a positive correlation between crevicular TNF- $\alpha$ and CAL and no other correlation between TNF- $\alpha$ and the rest of the periodontal parameters (9).

These results show that anti-TNF- $\alpha$ agents may influence the PD-related destructive processes (objectified by probing depth and clinical attachment level) and the gingival vascular status (objectified by gingival index and bleeding on probing index). Beneficial effects of Infliximab on marginal periodontal tissue is confirmed by Assuma et al in a study on primates as experimental models for PD. Anti-TNF- $\alpha$ therapy in combination with IL-1 antagonist inhibits $80 \%$ of the recruitment of inflammatory ce$11 \mathrm{~s}$ in the bone, osteoclast formation by $67 \%$ and bone loss $60 \%(9,10)$.

\section{CONCLUSIONS}

AS and PD are two conditions characterized by an altered inflammatory response of the patient, resulting in the destruction of the hard and soft connective tissue. Currently there are only three studies aiming to investigate the association between the two conditions, of which two have shown a higher prevalence of PD in patients diagnosed with AS, and periodontal disease more severe in patients with AS. These results suggest there might be an etio-pathogenic link between chronic PD and the developing of AS, a hypothesis that requires further studies in order to be demonstrated.

The present data indicate the need for closer collaboration between rheumatologists, periodontists and dental hygienists when treating patients with AS; importantly, treatment with anti-TNF- $\alpha$ agents may contribute to the improving of periodontal health. 


\section{REFERENCES}

1. Dumitriu S., Dumitriu H. Etiologia microbiana în parodontitele marginale cronice. Profilaxie si tratament antimicrobian, Editura Cermaprint, Bucureşti, 1996, ISBN:973-97483-2-5

2. Papapanou P.N. Periodontal diseases: epidemiology, Ann Periodontol, 1996;1:1-36

3. Popescu E.D., lonescu R. Compendiu de reumatologie, Editura Tehnică, 2002

4. Firesteain G., Budd R., Gabriel S. et al. Kelley's Textbook of Rheumatology, Elsevier, 2013, ISBN:9781437717389

5. Manakil J. Periodontal disease - A clinician's guide, 2012, InTech, ISBN 978-953-307-818-2

6. Pischon N., Pischon T., Gülmez E et al. Periodontal disease in patients with ankylosing spondylitis, Annals of the rheumatic diseases, 2010; 69:34-38
7. Keller J.J., Kang J.H., Lin H.C. Association between ankylosing spondylitis and chronic periodontitis: a population-based study, Arthritis and rheumatism, 2013; 65:167-173

8. Sezer U., Erciyas K., Pehlivan Y. et al. Serum cytokine levels and periodontal parameters in ankylosing spondylitis, Journal of periodontal research, 2012; 47:396-401

9. Mayer Y., Balbir-Gurman A., Machtei E.E. Anti-tumor necrosis factor-alpha therapy and periodontal parameters in patients with rheumatoid arthritis, Journal of periodontology, 2009; 80:1414-1420

10. Assuma R., Oates T., Cochran D. et al. IL-1 and TNF antagonists inhibit the inflammatory response and bone loss in experimental periodontitis, Journal of Immunology, 1998; 160:403-409 